\title{
Pulmonary embolism (acute or chronic)
}

\author{
Krivokuca I* \\ MC Zuiderzee Hospital, Lelystad, Netherlands
}

\begin{abstract}
\section{Introduction}

Pulmonary embolism (PE) and deep vein thrombosis (DVT) are known as venous thromboembolism (VTE). Blood clots in the deep veins of the legs (deep vein thrombosis) could break off and lodge in an artery in the lungs (pulmonary embolism) [1,2]. Rudolf Virchow postulated in 1856 that venous thrombosis could be initiated by abnormalities in the normal blood flow or stasis, increased hypercoagulability and vascular endothelial injury ("Virchow's triad.") [3]. It has been reported that the majority of patients $(84.1 \%)$ have complete resolution of the clots after 6 months of adequate anticoagulant therapy [4]. The extent and rapidity of recovery vary among different patients and different studies.
\end{abstract}

Pulmonary embolism (PE) and deep vein thrombosis (DVT) are known as venous thromboembolism (VTE). Blood clots in the deep veins of the legs (deep vein thrombosis) could break off and lodge in an artery in the lungs (pulmonary embolism). It has been reported that the majority of patients (84.1\%) have complete clots resolution after 6 months of adequate anticoagulant therapy. We briefly describe here a 66-year-old female with chronic dyspnea, due to recurrent pulmonary embolism and imaging diagnostic dilemma (acute or chronic residual pulmonary embolism), which we solved using the morphology of the embolus ('polo-mint sign').

\section{Case report}

A 66-year-old-woman, non-obese, ex-smoker with a history of COPD GOLD 1 and recurrent provoked pulmonary embolism had been treated for three weeks with tiotropium, on an outpatient basis. Despite that, she complained about progressive shortness of breath and mild fever. That is the reason why she was referred to our clinic for further evaluation. Her family history of venous thromboembolism was negative. She had no hormone replacement therapy or other risk factors for venous thromboembolism except a history of pulmonary embolism (when she was 31 and 39 years old). Furthermore, she had no anticoagulant therapy at the moment of presentation. The reason why she had no anticoagulant therapy was not clear.

\section{Findings}

At presentation, her body temperature was $37.4^{\circ} \mathrm{C}$, her respiratory rate was 20 breaths/min and her room air oxygen saturation was $92 \%$. The examination of the lungs revealed normal vesicular breath sounds, no wheezing or rhonchi. The remainder of her examination was completely normal.

\section{Diagnostic studies}

Laboratory test showed an elevated D-dimer level (d-dimer was 1715; normal $<130 \mu \mathrm{g} / \mathrm{l}$ ). Her serum $\mathrm{C}$ - reactive protein level was 219 (normal $<10 \mathrm{mg} / \mathrm{L}$ ) and her leucocyte count was 14.5 (normal $<10 \times$ $10^{9} / \mathrm{L}$ ). B-type natriuretic peptide (BNP) and troponin levels were not elevated. Arterial blood gas measurements revealed a respiratory alkalosis with hypoxemia ( $\mathrm{pH}$ was 7.52, a $\mathrm{PaCO}_{2} 19 \mathrm{~mm} \mathrm{Hg}$ and $\mathrm{PO}_{2}$ was $67 \mathrm{~mm} \mathrm{Hg}$ on room air). Her ECG was normal, with no signs of right heart strain and her chest radiography revealed no abnormalities. Computed tomography angiography (CTA) was performed and revealed pulmonary embolism and no pneumonia or an enlarged right ventricle. We did try to estimate the age of the embolus, using its morphology. This patient had previously two times pulmonary embolism and it is well known that some patients are unable to completely dissolve the clot. Although most of the emboli resolve spontaneously or due to treatment, it has been frequently reported in the literature that thrombotic residual could be seen after acute pulmonary embolism.

The question was, is the pulmonary embolism in this patient acute (new) or is that just a residual unresolved clot and that her symptoms are due to some other etiology, differential diagnosis involves, for instance, sinusitis or bronchitis, because of anamnestic fever and the elevated level of $\mathrm{C}$-reactive protein. However, because of the classic appearance of the "polo-mint sign" which is a CT finding in acute pulmonary embolism [5-7] (Figure 1), and no CT signs of pneumonia with elevated d-dimer, we decided to treat her pulmonary embolism. Her elevated level of C-reactive protein is probably caused by a combination of pulmonary embolism and recent sinusitis or bronchitis. The patient received the anticoagulant therapy, recovered slowly but uneventfully and left the hospital. Lifelong 'DOAC' (Direct Oral Anticoagulant) treatment was recommended.

\section{Discussion}

We used the CTA finding ("polo-mint" sign), in this patient, to estimate the age of the embolus. "Polo-mint" sign is a central filling defect surrounded by contrast (circumferentially) and it could be seen in patients with acute pulmonary embolism. Blood clots in the deep veins of the legs could break off and lodge in an artery in the lungs. It has been reported that the majority of patients $(84.1 \%)$ have complete resolution of the clots after 6 months of adequate anticoagulant therapy

${ }^{\star}$ Correspondence to: Krivokuca I, MC Zuiderzee Hospital Lelystad, Netherlands, E-mail: sciencia0@yahoo.com

Key words: acute pulmonary embolism, chronic pulmonary embolism, polo-mint sign, post-pe syndrome, residual thromboembolic obstruction

Received: December 02, 2019; Accepted: December 13, 2019; Published: December 17, 2019 


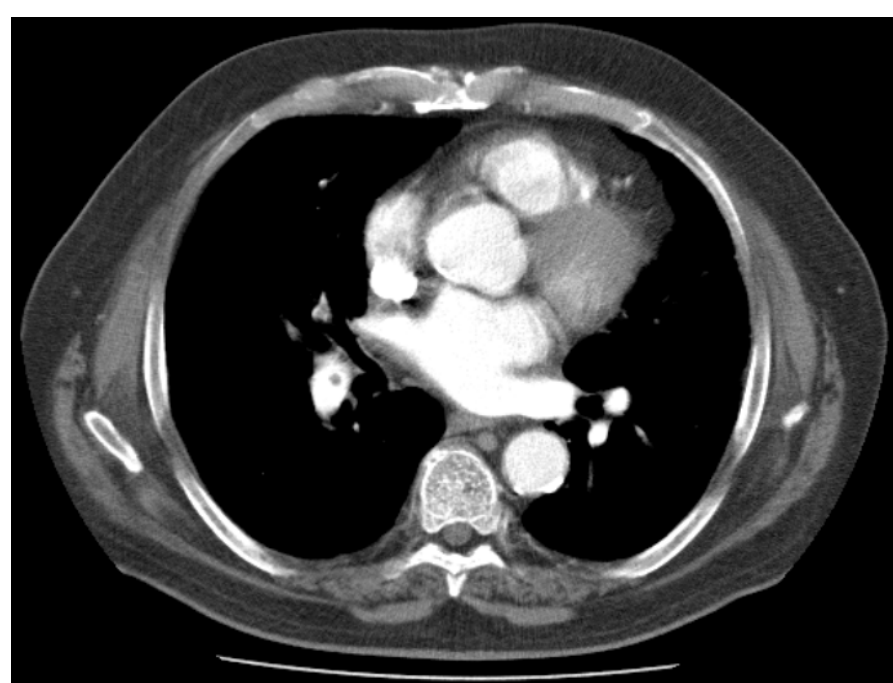

Figure 1. CTA Thorax with the classic appearance of the "polo-mint sign" (central filling defect surrounded by contrast).

[4]. The extent and rapidity of recovery vary among different patients and different studies.

Pulmonary embolism is a relatively common acute disorder with an annual incidence rate of 1-2 per 1000 patients with nonspecific symptoms and signs which is why pulmonary embolism remains a diagnostic challenge [8]. Recent studies suggest that up to $50 \%$ of survivors develop 'post-PE syndrome' which refers to persistent dyspnea, exercise intolerance, and impaired quality of life that persist for longer than 3 months after anticoagulant therapy for PE [9].

Anamnestic findings (pre-test probability), laboratory tests (D-dimer) and imaging (CTA) are very important parts of diagnostic algorithms for PE. CTA is one of the imaging tests for diagnosis of pulmonary embolism, which is available around the clock in most centres, very accurate and has a low rate of inconclusive results (35\%) [1]. Re-imaging and obtaining a new baseline after cessation of anticoagulant therapy, in patients with pulmonary embolism might be considered although that is currently not recommended [4].

Alternatives for CTA are ventilation-perfusion (V/Q) scintigraphy, V/Q spect (single-photon emission computed tomography) and pulmonary angiography. The ventilation-perfusion (V/Q) scintigraphy is a relatively not expensive, contrast agent sparing procedure and could be applied especially in patients with a normal chest X-ray, in patients with history of allergy to contrast agents, and in patients with severe renal failure but it is not readily available in all centres and it is frequently inconclusive (50\% of V/Q scintigraphies are inconclusive) [1]. V/Q spect, on the contrary, has the lowest rate of non-diagnostic tests $(<3 \%)$. Further, pulmonary angiography was the 'gold standard' for the diagnosis or exclusion of acute $\mathrm{PE}$, but it is not readily available in all centres and it is now not frequently performed (easy accessible CTA offers similar diagnostic accuracy) [1].

\section{CTA findings of acute and chronic pulmonary embolism}

CTA findings of acute and chronic pulmonary embolism could help clinicians to determine the age of the thrombus which could have therapeutic consequences when they consider starting anticoagulant therapy and when the optimal duration of anticoagulant therapy after PE has to be established.

\section{Acute pulmonary embolism findings are}

- Complete filling defect (vessel size normal or dilated).

- Eccentric filling defect with the acute angle with the artery wall.

- Central filling defect surrounded by contrast,

- 'Polo-mint sign' (in cross-section) [5-7].

- 'Railway track sign' (along the long axis of the vessel).

\section{Ancilliary finding}

Peripheral, wedge-shaped pure ground-glass opacity or groundglass and solid opacity together such as 'reversed halo sign' (infarct) [7]. 'Reversed halo sign' (atoll sign) is a nonspecific finding (central groundglass opacity surrounded by solid opacity) which could represent an infarct [7].

\section{Chronic pulmonary embolism findings are}

- Complete filling defect (vessel size normal or smaller than adjacent patent vessel).

- Peripheral, crescent-shaped defect with the obtuse angle with the artery wall.

- Web or flap (linear defect).

\section{Ancilliary finding}

- Mosaic perfusion pattern in the lungs.

- CT findings of pulmonary hypertension.

The laboratory tests such as highly sensitive, but non-specific d-dimer could improve interpretation of the CTA findings of acute and chronic pulmonary embolism. Acute pulmonary embolism laboratory finding is usually an elevated d-dimer level. Chronic pulmonary embolism finding is usually a normal d-dimer level (d-dimer levels after stopping an anticoagulant treatment could differ between different anticoagulant therapies such as DOAC or warfarin) [10].

The persistent obstruction of pulmonary arteries by organized chronic thrombi, could lead to CTEPH (chronic thromboembolic pulmonary hypertension), with a cumulative incidence of 0.1 to $9.1 \%$ [1]. If this 66-year-old symptomatic female patient did not have a 'polo-mint sign' but signs of residual pulmonary obstruction, such as a peripheral, crescent-shaped defect (with the obtuse angle with the artery wall), web or flap with the CT findings of pulmonary hypertension (mosaic perfusion pattern in the lungs) then a different diagnostic and therapeutic approach would be chosen (then it would be necessary to measure the mean pulmonary artery pressure, which should be lower than $25 \mathrm{mmHg}$ to exclude CTEPH).

\section{Conclusion}

Clinicians could use the laboratory tests, anamnestic findings and also the morphology of the embolus (CTA findings) to determine the age of embolus (acute or chronic) when they consider starting anticoagulant therapy or when they try to establish the optimal duration of the anticoagulant therapy.

\section{References}

1. Konstantinides SV, Meyer G, Becattini C, Bueno H, Geersing GJ, et al. (2019) 2019 ESC Guidelines for the diagnosis and management of acute pulmonary embolism developed in collaboration with the European Respiratory Society (ERS). Eur Heart $J$ pii: ehz405. [Crossref] 
2. Wells PS (2007) Integrated strategies for the diagnosis of venous thromboembolism. $J$ Thromb Haemost 1:41-50. [Crossref]

3. Mukhopadhyay S, Johnson TA, Duru N, Buzza MS, Pawar NR, et al. (2019) Fibrinolysis and Inflammation in Venous Thrombus Resolution. Front Immunol 10: 1348. [Crossref]

4. den Exter PL, van Es J, Kroft LJ, Erkens PM, Douma RA, et al. (2015) Thromboembolic resolution assessed by CT pulmonary angiography after treatment for acute pulmonary embolism. Thromb Haemost 114: 26-34. [Crossref]

5. Shepard JO (2018) Thoracic imaging. The Requisites E-Book. Chapter 11. Elsevier Health Sciences.

6. Wittram C, Maher MM, Yoo AJ, Kalra MK, Shepard JA, et al. (2004) CT angiography of pulmonary embolism: diagnostic criteria and causes of misdiagnosis. Radiographics 24:1219-1238. [Crossref]
7. Singh A (2017) Emergency radiology: Imaging of acute pathologies, Springer. 23: 390

8. Naess IA, Christiansen SC, Romundstad P, Cannegieter SC, Rosendaal FR, et al (2007) Incidence and mortality of venous thrombosis: a population-based study. $J$ Thromb Haemost 5: 692-699. [Crossref]

9. Tavoly M, Wik HS, Sirnes PA, Jelsness-Jorgensen LP, Ghanima JP, et al. (2018) The impact of post-pulmonary embolism syndrome and its possible determinants. Thromb Res 171: 84-91. [Crossref]

10. Legnani C, Martinelli I, Palareti G, Ciavarella A, Poli D, et al. (2019) D-dimer levels during and after anticoagulation withdrawal in patients with venous thromboembolism treated with non-vitamin K anticoagulants. PLoS One 14: e0219751. [Crossref]

Copyright: (C2019 Krivokuca I. This is an open-access article distributed under the terms of the Creative Commons Attribution License, which permits unrestricted use, distribution, and reproduction in any medium, provided the original author and source are credited. 